\title{
La reunión como herramienta de gestión del desarrollo educacional
}

\author{
Andrés Saavedra Castellanos ${ }^{1}$
}

Recibido 10 de agosto de 2019 · Aceptado 01 de noviembre de 2019

\section{RESUMEN}

El artículo aborda las bases teóricas de la reunión. Su novedad radica en la presentación de los elementos a tener en cuenta para convertirla en una herramienta de gestión del desarrollo educacional. En tal sentido, se definen los niveles de participación que tienen lugar en las reuniones, según el tipo y forma de realización. Unido a ello, se precisan las relaciones que deben establecerse entre participación, potenciación de competencias profesionales y acuerdos.

Este trabajo es producto de la experiencia del autor en la dirección de proyectos de investigación, asesorías y capacitaciones desarrolladas con docentes y directivos en Cuba y Latinoamérica. Es un punto de vista que se pone a consideración de investigadores, docentes, especialistas y profesionales para su debate y discusión.

Palabras clave: reunión, participación, competencias y acuerdos.

\section{Meetings as a Management Tool for Educational Development}

\section{ABSTRACT}

This article address the theoretical foundations of meetings. Its new proposal lies in the presentation of the elements to consider in order to transform meetings into a management tool for educational development. In this sense, the levels of participation in meetings are defined according to type and form. In conjunction with this, the author outlines the relationships that must be established between participation, strengthening of professional skills and agreements.

This work is the product of the author's work in the leadership of research projects, advisory and teacher and leader training in Cuba and Latin America. It is a perspective offered up for debate and discussion by researchers, teachers, specialists and professionals.

Key words: meeting, participation, competitions, agreements.

Doctor en Ciencias Pedagógicas. Profesor Titular de la Universidad de Granma, Cuba. asaavedrac@udg.co.cu 


\section{Introducción}

Los complejos procesos de cambio y transformaciones que acontecen en la actualidad, en el orden económico, político y social, imponen el reto de gestionar el desarrollo educacional sobre bases científicas. Para ello se deben tomar decisiones objetivas, que permitan dar respuestas a los problemas que enfrentan las instituciones educacionales. En tal sentido, la reunión puede constituirse en una herramienta fundamental si se utiliza adecuadamente.

Sobre las reuniones existen tantos estudios como investigadores han escrito al respecto. Sus principales aportes han girado en torno al papel de los profesionales responsabilizados con su planificación, conducción y seguimiento. Sin embargo, no son estas las únicas variables a tener en cuenta para el logro de los objetivos organizacionales e individuales. Al referirse a las reuniones, Gandarilla (20I I) expresó "no son un fin en sí mismas, sino una herramienta, una técnica, un medio de trabajo, y como tal debemos usarlas" (p. 2).

En este artículo se parte por presentar un estudio realizado en diversas instituciones educacionales latinoamericanas. Se precisan los niveles de participación a tener en cuenta en los tipos y formas de reuniones existentes, se define el concepto de la reunión como herramienta del desarrollo educacional, en relación con la participación y la potenciación de competencias profesionales.

Es producto de la experiencia teórica y práctica del autor, al desempeñarse, durante más de 20 años como investigador, jefe de departamento, vicedecano, decano, vicerrector de la Universidad de Granma y asesor de directivos educacionales, empresariales, de instituciones hospitalarias, del sector público y privado, en Cuba y Latinoamérica.

\section{La participación como elemento esencial de la reunión}

Al referirse a las reuniones, Carnota (I99I) precisa que es una actividad legítima que resulta insustituible para el logro de objetivos, en las que la inteligencia colectiva, la necesidad de cooperación, el 
comprometimiento con la ejecución, el surgimiento de ideas o el intercambio de conocimientos son factores fundamentales.

En sentido general, se ha entendido la reunión como un encuentro entre dos o más personas, con la finalidad de intercambiar informaciones $\mathrm{y} / \mathrm{o}$ analizar problemas para la toma de decisiones. Este encuentro se ha concebido, por lo general, con la presencia física de sus participantes, en un mismo lugar y tiempo determinado.

De igual manera, se enfatiza que la preparación, dirección y seguimiento, por los organizadores o conductores de la reunión, son determinantes en los resultados que se pretenden alcanzar. Si bien estas variables son importantes, existen otras que se debe tener en cuenta.

Con la finalidad de constatar las variables que inciden en el desarrollo de las reuniones, se hicieron IOO observaciones a organizaciones educacionales, culturales, deportivas, económicas, políticas, gubernamentales y hospitalarias, en Cuba y Latinoamérica, en la etapa de enero de 2013 a diciembre de 2018, complementadas con entrevistas.

Las observaciones se hicieron sobre la base de los indicadores: hora de inicio-conclusión, condiciones físicas, psicológicas y materiales que mediatizan las reuniones, competencias mostradas por los conductores y organizadores, análisis de los puntos de la agenda, conflictos generados en los debates y adopción de los acuerdos, en lo fundamental.

En el $80 \%$ de las reuniones observadas se pudo constatar como regularidades las siguientes:

- Iniciaron y concluyeron aproximadamente a la hora prevista.

- Ambiente físico, psicológico y emocional adecuado.

- Los participantes tuvieron la oportunidad, en su mayoría, de expresar sus puntos de vistas.

- Consenso en los acuerdos adoptados.

En el $20 \%$ de las observaciones restantes se constató, indistintamente:

- Iniciaron con más de 30 minutos de atrasos o concluyeron pasada una hora o más de lo previsto. 
- Falta de preparación de los conductores de la reunión.

- Personas convocadas sin previo conocimiento de los objetivos.

- Falta de compromiso de sus participantes con los puntos tratados, expresado en la desviación de su atención a otras actividades, como atender el móvil, salidas del local en el que se estuvo desarrollando la reunión, conversación con otros participantes mientras se dirige el punto por el conductor, en lo fundamental.

- Desacuerdos y conflictos, irreconciliables, durante el debate de determinados puntos de la agenda o en la propuesta de acuerdos.

Los resultados de la observación se complementaron con entrevistas al 30\% de los participantes, en las 100 reuniones observadas. La muestra fue intencional, atendiendo a la disponibilidad de los mismos para acceder a la entrevista.

Las preguntas estuvieron dirigidas a evaluar las causas del nivel de satisfacción e insatisfacción experimentado en las diversas reuniones a las que han sido convocados en su vida profesional.

Como regularidad, en los planteamientos se pudo constatar que la satisfacción experimentada en diversas reuniones estuvo dada en:

- El conocimiento previo de los objetivos.

- Previa socialización de la agenda y puntos a tratar.

- Los debates y aportes que se generaron.

- El clima emocional favorable.

- La satisfacción de las necesidades organizacionales e individuales.

- Las habilidades y competencias mostradas por los conductores y organizadores de la reunión.

Al referirse a la insatisfacción experimentada, coincidieron en exponer de manera negativa los puntos antes expresados. A ellos agregaron los siguientes:

- La urgencia con la que se les citaron.

- Imposiciones de acuerdos tomados por los conductores.

- Falta de consenso en la toma de decisiones.

- Poca o nula posibilidad de exponer sus puntos de vista. 
Los resultados de los instrumentos antes descritos permitieron determinar diversas variables que inciden en el desarrollo exitoso de una reunión. Entre estas se puede mencionar:

- Las condiciones en que transcurre la reunión: horario, duración de los puntos de la agenda, las condiciones físicas e higiénicas del lugar de trabajo.

- El estilo de dirección que asumen sus conductores, expresado en el ambiente emocional que se genera.

- La percepción y valoración por cada profesional de la funcionalidad que tiene la reunión para satisfacer sus necesidades individuales y organizacionales.

- La posibilidad de exponer puntos de vistas e ideas para consensuar acuerdos.

- Retroalimentación y reconocimiento, concretado en el grado de aceptación o rechazo de los puntos de vista e ideas que se exponen.

Estas variables, dinamizadas por la percepción de los problemas individuales, grupales y organizacionales, incidieron en los niveles de participación de los presentes en la reunión. Según sea la disposición de los miembros de la institución educacional a participar en la reunión, así será la calidad del cumplimiento de los objetivos propuestos. El término "participar", en su acepción más general, significa ser, tomar o tener parte, intervenir, colaborar, involucrarse en determinados asuntos.

De aquí que la participación en las reuniones se defina como la acción emprendida por los miembros de la institución educacional que les permite sentir y/o tomar parte de la reunión, al asumir una acción activa de identificación de problemas, formulación de propuestas de soluciones, negociación de acuerdos o una acción pasiva de apoyo a las decisiones tomadas por otros, con las cuales está de acuerdo.

Desde la perspectiva de dicha definición se puede identificar, en lo fundamental, dos niveles de participación:

I. La participación acción pasiva.

2. La participación acción activa. 
Se está en presencia de miembros con participación acción pasiva cuando estos se sienten parte de la reunión; sin embargo, su accionar se limita a estar presentes. No consideran necesaria su intervención, en ninguno de los puntos tratados; no obstante, apoyan las decisiones acordadas por todos o la mayoría de los participantes, sin objetar.

Se está en presencia de miembros con participación acción activa cuando estos no solo se sienten parte de la reunión, sino que también se involucran, al intervenir activamente en uno o más puntos de la agenda, identifican problemas, formulan propuestas de soluciones y negocian acuerdos.

En ambos niveles media la satisfacción de determinadas necesidades, que impulsan a los miembros de la institución educacional a participar en las reuniones convocadas. Estas necesidades representan el estado de carencia, expresado en la representación de la situación actual y la aspiración de una situación deseable, en el que la reunión constituye un medio que contribuye al logro de tal aspiración.

Sobre las necesidades de las personas existen diversas teorías, entre ellas Maslow (1954) postula que cada persona tiene una jerarquía de cinco necesidades:

a) Fisiológicas: protección, salario, vivienda, alimento, agua, temperatura adecuada, etc.

b) De seguridad: ambiente seguro de trabajo, normas definidas con claridad, ausencia de amenazas y coerción o trato arbitrario, etc.

c) Sociales: sentimiento de pertenencia a la organización, vinculación social, interacción, amistad, afecto, amor, etc.

d) Estima: autoestima, deseo de logro, estatus y reconocimiento externo.

e) Autorrealización: Ser lo suficientemente competente de forma sostenida, búsqueda de nuevas responsabilidades y metas.

De la jerarquía precisada por Maslow (I954), se destaca que es posible que estén activas dos o más necesidades simultáneamente. De aquí que las condiciones físicas en las que se desarrolla la reunión, expresadas en condiciones de iluminación, temperatura, mobiliario, estilos de conducción democrático o coercitivo y amenazador, 
tienen su incidencia en la satisfacción de necesidades fisiológicas y de seguridad. De igual manera, los sentimientos de pertenencia a la institución educacional, la interacción entre los participantes, las competencias, el compromiso y la responsabilidad con las metas educacionales tiene su incidencia en la satisfacción de necesidades sociales, estima y autorealización.

La satisfacción de las necesidades de los miembros de una institución educacional participantes en una reunión varía de persona a persona, si se tiene en cuenta que sus capacidades, competencias profesionales son diferentes y varían con el tiempo. No obstante esas diferencias, al reunirse en grupo manifiestan patrones de comportamientos más o menos semejantes, dinamizados por necesidades, en cierta medida, comunes.

Si bien los niveles de participación en una reunión están condicionados por las necesidades expresadas anteriormente, los tipos de reuniones que se convocan van a permitir una participación acción activa o pasiva.

\section{Tipos de reuniones en relación con los niveles de participación. La potenciación de competencias profesionales}

De acuerdo con los objetivos a cumplir, se convocan reuniones de diferentes tipos, las cuales pueden clasificarse según el contenido y la forma de interacción.

Por su contenido, la reunión se puede clasificar en:

a) Reunión informativa.

b) Reunión de análisis y propuesta de soluciones a problemas.

c) Reunión formativa.

d) Reunión mixta.

Por la forma de interacción:

Según la estabilidad temporal y estructural:

a) Reunión sistemática o cíclica: ejemplo de ella se puede mencionar la reunión del Consejo de Dirección, Junta Directiva, Consejo científico-técnico, entre otras. 
b) Reunión ocasional: Despachos, entrevistas, mitines, actos públicos, asambleas, entre otras.

c) Por las formas presenciales de interacción:

i. Presencial: aquellas en las que todos sus miembros comparten un mismo espacio físico y tiempo.

ii. Semipresencial: Por ejemplo video-reuniones, telellamadas, el uso de plataformas online, entre otras.

Esquema $\mathrm{n}^{\circ} \mathrm{I}$. Tipos de reuniones por el contenido y la forma:

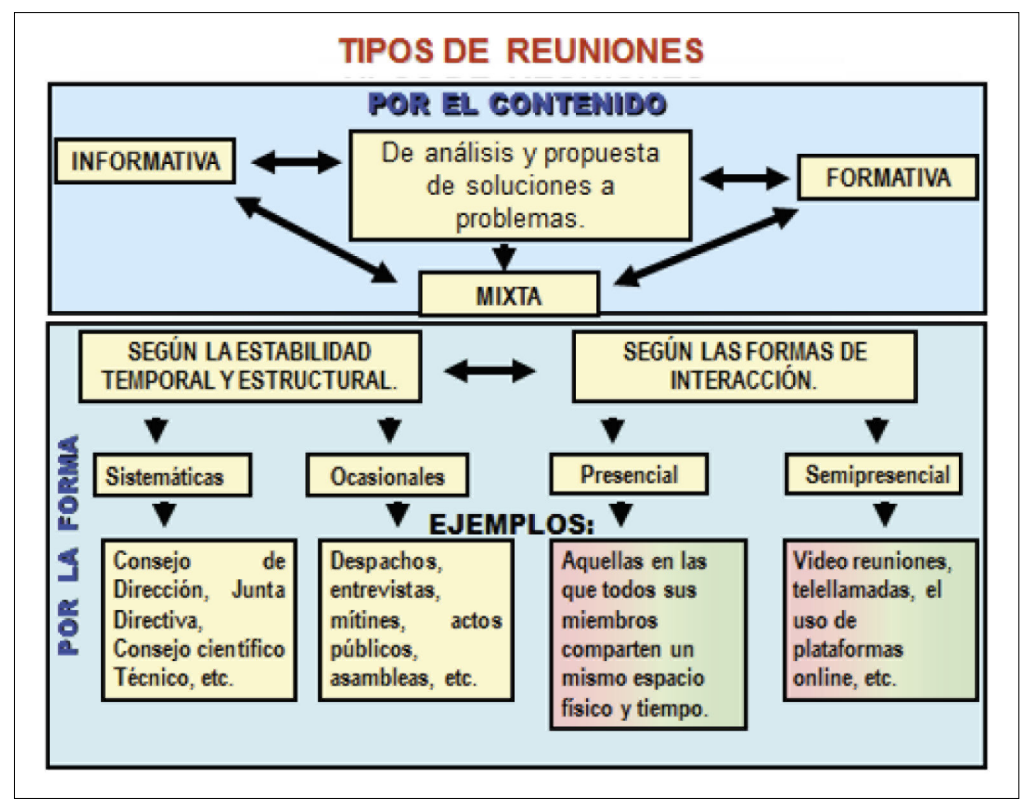

Fuente: elaboración propia (2019).

Para una mejor comprensión de los tipos de reuniones, a continuación se explica en qué consisten, así como las combinaciones que pueden existir por su contenido y forma de interacción.

a) La reunión informativa es convocada para informar decisiones que inciden directa o indirectamente en el cumplimiento de las funciones de los convocados. También es utilizada para recoger alguna información necesaria. La misma no siempre requiere de una agenda estructurada formalmente con determinados puntos. 
Este tipo de reunión se caracteriza, en general, por ser muy breve. Sin embargo, no siempre es así. Tal condición va a depender de un grupo de factores que pueden influir en su durabilidad:

- El número de personas participantes. A mayor número de participantes, es posible, mayor número de intervenciones.

- Volumen y complejidad de la información: a mayor comprensión de la información que se brinda, menor es el número de intervenciones para su aclaración.

- Habilidades comunicativas y estado emocional que genera el conductor de la reunión: a mayor conocimiento de la información, exposición clara por parte del conductor de la reunión, así como la generación de un ambiente emocional positivo, menos resistencia e intervenciones de los participantes.

La reunión informativa puede combinarse con las formas expresadas en el esquema $n^{\circ} I$.

b) La reunión de análisis y propuesta de soluciones a problemas: es convocada para analizar determinados problemas que enfrenta la institución educacional, la generación de posibles soluciones que se convierten en decisiones (acuerdos) que deben enfrentar sus miembros, en función de gestionar el desarrollo organizacional.

Para su desarrollo se sugiere, siempre que se pueda, definir previamente su agenda. Este es un documento en el que aparecen recogidos, en lo fundamental, lugar y hora de realización, el objetivo u objetivos de la reunión, los temas o puntos a tratar, tiempo de duración aproximado de cada punto, así como los responsables de su conducción. Debe socializarse para que los convocados puedan prepararse.

Esta reunión requiere que todos o la mayoría de sus participantes se manifiesten de manera activa en los análisis de los problemas y en la propuesta de soluciones.

c) La reunión formativa: tiene como objetivo formar y desarrollar en los miembros de la institución educativa determinadas competencias necesarias para la gestión del desarrollo educacional, para lo cual deben sostener una participación acción activa. 
Puede formar parte de la reunión de análisis y solución de problemas, siempre que tengan un enfoque consciente de potenciar las competencias de los participantes. De igual manera, según la forma, se puede desarrollar a través de las reuniones sistemáticas u ocasionales.

Sin embargo, las reuniones formativas siempre coinciden con las distintas formas de capacitación, superación profesional y formación académica, tales como la conferencia especializada, el seminario, el taller, el debate científico, el curso, el entrenamiento, el diplomado, la especialidad, la maestría, el doctorado, entre otras, que posibilitan el estudio y la divulgación de los avances del conocimiento, la ciencia, la tecnología y el arte.

d) La reunión mixta se combina con dos o más tipos y formas de las expresadas en este artículo.

e) La reunión sistemática o cíclica: aquella que, por su nivel de estabilidad estructural y funcional, está normada en su finalidad y cantidad o tipo de participantes. Tiene un carácter cíclico porque no se trata de discutir puntos que por su redacción pueden semejarse a los analizados en reuniones anteriores, sino más bien por abordar los niveles de desarrollo alcanzado en cada nueva etapa o ciclo. Sus miembros deben mantener una participación acción activa. Por ejemplo, la reunión del Consejo de Dirección, Junta Directiva, Consejo científico-técnico, entre otras.

f) La reunión ocasional: aquella en la que no se norma su composición estructural y funcional. Se convoca en correspondencia con las situaciones que se presentan en un momento determinado. Estas situaciones pueden ser generadas por un problema urgente al que haya que buscar una solución inmediata, la necesidad de transmitir o recopilar determinada información, la reafirmación o promoción de ideas y actores políticos, entre otras. Puede ser convocada por cualquier miembro de la institución educacional, independientemente de su estatus profesional. La participación de los convocados puede ser activa o pasiva. Por ejemplo, los despachos, entrevistas, mitines, actos públicos, asambleas, entre otras. 
g) Reunión presencial: aquella que se desarrolla con la presencia de sus miembros en un mismo espacio físico y tiempo determinado. Implica el contacto cara a cara.

h) Reunión semipresencial: No requiere la presencia de sus miembros en un mismo espacio físico. Se necesita, de ser posible, que coincidan en un mismo tiempo conductores y participantes para que exista la interactividad inmediata. Por su contenido puede ser informativa, de análisis y solución de problemas, formativa o mixta. Su uso más frecuente es en instituciones en las que sus miembros se encuentran distantes, así como en la comunicación entre miembros de distintas organizaciones, con objetivos comunes a alcanzar. Ejemplo de ellas: video-reuniones, telellamadas, el uso de plataformas online, entre otras.

Conocer los tipos de reuniones es de gran importancia para organizadores, conductores y participantes. Su conocimiento permite elegir la forma más conveniente de realización según la situación que se debe afrontar, las necesidades de los participantes potenciales y las exigencias del momento, en aras de gestionar las formas de participación que deben prevalecer y el desarrollo de la institución educacional.

Para que los tipos de reuniones, antes descritas, constituyan una herramienta de gestión del desarrollo de la institución educacional, se deben relacionar los niveles de participación con la potenciación de competencias profesionales de los implicados.

En el contexto de este artículo se define "competencia profesional" como el sistema de conocimientos, habilidades, actitudes, aptitudes, capacidades y valores adquiridos por directivos y miembros de la institución educacional, en un proceso de formación permanente, aplicables al ejercicio de las funciones adjudicadas. Se expresa en el cumplimiento, con calidad, de los objetivos individuales y organizacionales propuestos y en la ventaja competitiva alcanzada.

La ventaja competitiva se concreta no solo en la diferenciación de cada miembro y de la institución educacional, en comparación con otros $\mathrm{u}$ otras, respecto al saber, saber hacer y los valores que los caracterizan, durante el ejercicio de determinadas funciones; sino en 
la superación permanente de las capacidades con las que cuentan, el perfeccionamiento de sus modos de actuación profesional para el cumplimiento, con calidad, de los objetivos propuestos.

Sobre esta base, se define a la reunión como herramienta de gestión del desarrollo de la institución educacional, la que propicia la necesaria participación e interacción entre sus miembros, en función de la complementación y potenciación de competencias profesionales que contribuyan al logro de los objetivos institucionales e individuales propuestos, con mayor ventaja competitiva.

\section{Conclusiones}

- Los niveles de participación de los miembros de una institución educativa constituye un elemento esencial a tener en cuenta por organizadores y conductores de la reunión, en tanto aportan las premisas claves para el cumplimiento, con mayor calidad, de los objetivos propuestos.

- Determinar adecuadamente el tipo de reunión a desarrollar por su contenido y forma, tomando como base los niveles de participación, en relación con la potenciación de las competencias profesionales, permite gestionar el desarrollo de la institución educacional.

\section{Referencias bibliográficas}

Carnota, O. (199I). Cuando el tiempo no alcanza. La Habana: Editorial de Ciencias Sociales.

Gandarilla, A. (20I I). Las reuniones y despachos. En soporte electrónico. Conferencia impartida al Consejo de la Administración Provincial en Granma. Bayamo, Cuba.

Maslow, A. H. (1954). Motivation and Personality. New York: Harper and Row.

Saavedra, A. (2017). El liderazgo en la gestión educacional latinoamericana. Revista Gestión y Región Universidad Católica de Pereira, 24.

Saavedra, A. (2017). El liderazgo directivo en la gestión del desarrollo organizacional. OLIMPIA. Revista de la Facultad de Cultura Física de la Universidad de Granma, I5(52). 